\title{
Zwischen Effizienz und Suffizienz
}

\author{
Griffige Konzepte erhöhen die öffentliche Akzeptanz von Umweltpolitik. Öko-Effi- \\ zienz ist ein pragmatischer Ansatz, der die strategischen Möglichkeiten betont, \\ Umweltbelastungen ohne wirtschaftliche Eimbußen zu verringern. Insofern bildet \\ dieses Konzept den Gegenpol zu einer Suffizienz-Strategie, die letztlich ökologi- \\ sche Grenzen des Wachstums postuliert. Öko-Effizienz und Suffizienz lassen sich \\ allerdings bei näherer Betrachtung insbesondere im Konsumbereich kaum klar \\ abgrenzen. Daher sollten im Mitfelpunkt der Diskussion statt dieser Polarisierung \\ tragfähige Konzepte für einen ökologischen Strukturwandel stehen.
}

Ö Von Jochen Diekmann ko-Effizienz kann allgemein als Effizienz definiert werden, mit der ökologische Ressourcen zur Befriedigung menschlicher Bedürfnisse genutzt werden (1). In diesem allgemeinen Sinne könnte Öko-Effizienz als eine notwendige Bedingung rationaler Ressourcenverwendung angesehen werden. Als operationale Indikatoren der Öko-Effizienz werden aber gewöhnlich Output-Input-Relationen verwendet, die z.B. die Produktion eines Gutes auf die hierdurch verursachten Schadstoffemissionen beziehen. Dementsprechend steht z.B. die Minimierung der Material- bzw. Energieintensität von Gütern und Dienstleistungen als Kriterium für Öko-Effizienz im Vordergrund. Eine solche Minimierung von spezifischen Faktorverbräuchen kann jedoch aus umweltökonomischer Sicht vor allem dann problematisch sein, wenn dies mit hohen (Opportunitäts-) Kosten verbunden ist. Insofern kann ein Konflikt zwischen so verstandener Öko-Effizienz und ökonomisch effizienter Ressourcenverwendung nicht ausgeschlossen werden (2). Vor diesem Hintergrund bestehen Vorbehalte dagegen, (technisch definierte) ÖkoEffizienz als allgemeine Leitlinie der Umweltpolitik zu akzeptieren.

Effizienz-Strategien werden häufig damit begründet, daß große Potentiale zur Ressourceneinsparung bestehen, die ohnehin (d.h. auch abgesehen von ökologischen Überlegungen) rentabel sind - aufgrund von unterschiedlichen Hemmnissen aber bisher nicht ausgeschöpft wurden. So können sich bestimmte Maßnahmen zur Steigerung der Energieeffizienz schon deshalb lohnen, weil beträchtliche Energiekosten eingespart werden. Aus ökonomischer Sicht haben solche ,win-win"-Optionen selbstverständlich Priorität. Angesichts der hohen Anforderungen an die ökologische Wirksamkeit z.B. zur Erreichung der klimapolitischen Ziele dürften solche (kostenlosen) Maßnahmen allein aber kaum ausreichen.

Während Öko-Effizienz im Sinne der Minimierung von spezifischen Umweltintensitäten in den betroffenen Bereichen zu weit ginge, würde eine alleinige Konzentration auf solche Umweltschutzmaßnahmen, die sich ohnehin (aus anderen Gründen) lohnen, zu kurz greifen.

\section{Drei Komponenten der ökologischen Entwicklung}

Das Paradigma der Öko-Effizienz richtet den Fokus auf die Intensität der Ressourcennutzung. Dabei werden unter Umständen andere Komponenten der ökologischen Entwicklung zu sehr ausgeblendet. Aus ökologischer Sicht ist letztlich der absolute Ressourcenverbrauch (RV) maßgeblich, der sich vereinfachend in die folgenden Faktoren zerlegen läßt:

\section{$\boldsymbol{R V}=$ Intensität $\cdot$ Struktur $\cdot$ Aktivität .}

Diese Komponentenzerlegung, die vor allem bei Indikatoranalysen für den Industriebereich verwendet wird, aber auch auf Haushalte anwendbar ist, macht deutlich, daß als strategische Ansatzpunkte zur Verminderung des Ressourcenverbrauchs neben der Intensitätskomponente ebenso Struktureffekte und das Niveau wirtschaftlicher Aktivitäten zu betrachten sind.

In diesem Zusammenhang hängt das Verhältnis von Effizienz und Suffizienz davon ab, wie eng die Begriffe definiert werden: Wird Effizienz in einem engen Sinne als Output-Input-Relation verstanden, dann fuihrt dies zu einer beschränkten Betrachtung auf einzelwirtschaftliche oder technische Intensitäten, während sich der Suffizienz-Ansatz im engeren Sinne auf die Aktivitäts- komponente reduziert. In diesem Fall wäre der ökologische Strukurwandel eine von beiden Konzepten unbeachtete Restgröße.

Werden hingegen beide Begriffe in einem weiteren Sinne verstanden, kann es zu mehr oder weniger bedeutsamen Überlappungen der strategischen Ansätze kommen, die Fragen der begrifflichen Abgrenzung von Effizienz und Suffizienz aufwerfen. Solche Abgrenzungsfragen erstrecken sich insbesondere auf den Bereich der Strukturveränderungen, d.h. auf die Substitutionsmöglichkeiten bei der Produktion bzw. dem Konsum von Gütern und Dienstleistungen.

\section{Abgrenzungsprobleme}

Wenn Öko-Effizienz als prioritäres ökologisches Strategiekonzept tragfähig sein soll, dann ist es erforderlich, den Effizienzbegriff auch in Bereichen der unmittelbaren Bedürfnisbefriedigung, also in privaten Haushalten sowie im Personenverkehr, klar von Elementen einer SuffizienzStrategie abzugrenzen. Dies ist allerdings schon deswegen äußerst schwierig, weil hierzu letztlich die Bedürfnisse der Menschen definiert werden müssen. Es dürfte allenfalls möglich sein, sich auf bestimmte menschliche Grundbedürfnisse im Sinne eines (kulturell determinierten) Existenzminimums zu einigen. Darüber hinaus sind allerdings (insbesondere in einer ,Wohlstandsgesellschaft") kaum generalisierende Aussagen möglich, da die persönliche Bedürfnisbefriedigung - anders als der Produktionsoutput von Industrieunternehmen - von individuellen Situationen und Wertungen abhängt.

So kann z.B. eine konkrete Maßnahme wie CarSharing in Abhängigkeit von der relevanten Bedïrfniskategorie entweder als Effizienzmaßnahme bewertet werden (Mobilität als Bedürfnis) oder aber die Suffizienz betreffen (Autobesitz als Bedürfnis) (3). Eine Begrenzung der Höchstgeschwindigkeit z.B. auf Tempo 100 km/h würde Energieverbrauch und Emissionen senken, wobei sich die Fahrtzeit häufig nur wenig verlängern würde und sich gleichzeitig die Unfallgefahr vermindern wïrde; dennoch wird diese Einsparmöglichkeit offensichtlich nur von wenigen Autofahrern freiwillig genutzt. Eine Absenkung der Raumtemperatur von 22 auf 18 Grad kann für manche Menschen eine win-winwin-Maßnahme sein (Umweltentlastung, Energiekosteneinsparung, höheres Wohlbefinden), während dies für andere einen einschneidenden Verzicht bedeuten würde. Selbst Wärmedämmstandards, die sich bei normalem Lüftungsver- 


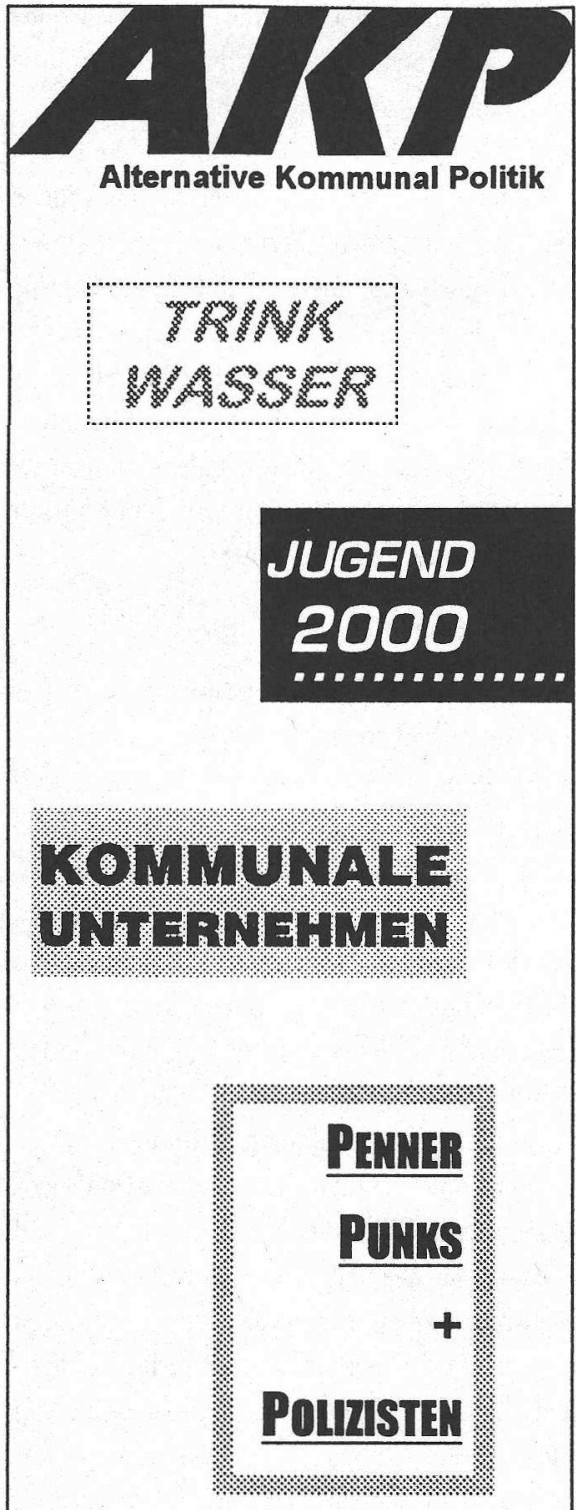

...diese Schwerpunkt-Themen der letzten AKP-Ausgaben zeigen die breite Palette der Fachzeitschrift „Alternative Kommunalpolitik". Sie ist ein unverzichtbarer Informationsdienst über alle Bereiche bündnisgrüner Kommunalpolitik. Jedes Heft enthält zusätzlich aktuelle Nachrichten und Hintergrundberichte. Wichtige Themen werden in Büchern und Sonderheften vertieft.

Probeheft und Gesamtkatalog anfordern bei:

Alternative Kommunal Politik

Luisenstr. 40, 33602 Bielefeld Ruf 0521/177517, Fax 0521/177568

E-Mail: AKP-Redaktion@t-online.de Internet: www.gruene.de/akp-redaktion halten einzelwirtschaftlich rentieren, können individuell anders bewertet werden, wenn ein besonders starkes Bedürfnis nach frischer Luft besteht.

Solche Beispiele zeigen, daß nicht alle Maßnahmen zur Ressourceneinsparung - unabhängig davon, ob sie als sinnvoll angesehen werden eindeutig in die Kategorien Effizienz und Suffizienz eingeteilt werden können. Eine solche Unterteilung ist deshalb als Ausgangspunkt ciner Strategieformulierung nur begrenzt hilfreich.

Wichtiger als eine (gekünstelte) Trennung zwischen Effizienz- und Suffizienz-Anpassungen ist die Frage nach der relativen Bedeutung der unterschiedlichen Determinanten von Umweltverhalten. Ein besseres Verständnis des Zusammenwirkens von technologischen, ökonomischen, soziologischen und sozialpsychologischen Effekten könnte insbesondere dazu beitragen, geeignete politische Instrumente für den Umweltschutz zu identifizieren. Es dürfte inzwischen unstrittig sein, daß Umweltverhalten sowohl durch Randbedingungen, durch ökonomische Faktoren als auch durch allgemeine und spezielle Einstellungen bestimmt wird. Dies spricht immerhin für die Vermutung, daß eine entsprechende Bündelung unterschiedlicher Instrumente wirkungsvoller ist als eine Beschränkung allein auf eine Instrumentenart. Eine Kampagne, die sich allein am Begriff der Öko-Effizienz orientiert, könnte mißverstanden werden als Aufruf, Umweltverhalten auf Situationen $\mathrm{zu}$ beschränken, in denen keine nennenswerten individuellen Anstrengungen erforderlich sind. Sie könnte die Aufmerksamkeit außerdem zu sehr auf die Anschaffung technisch effizienter Geräte oder Fahrzeuge lenken, statt auf das Verhalten bei deren Nutzung. In diesem Zusammenhang ist auch danach zu fragen, wie eine Betonung von öko-Effizienz auf das allgemeine Umweltbewußtsein wirkt.

\section{Ökologischer Strukturwandel entscheidend}

Öko-Effizienz und Suffizienz sind unscharfe Konzepte, die (scheinbar) unterschiedliche Wege zu einer nachhaltigen Entwicklung weisen: einen bequemen, der - sobald gefunden - leicht beschritten werden kann, und einen steinigen, der Opfer verlangt. Es sind aber Zweifel angebracht, ob wirklich eine Wahl zwischen solchen Wegen (,Königsweg oder Sackgasse“) besteht; es deutet vielmehr einiges darauf hin, daß es sich um Teilstrecken eines gemeinsamen, langen Weges handelt.
Akzeptiert man die oben skizzierte Komponentenbetrachtung der ökologischen Entwicklung, dann kommt eine wesentliche dritte Teilstrecke hinzu, nämlich die des ökologischen Strukturwandels, der im Spannungsverhältnis steht zwischen Effizienz und Suffizienz. Der Begriff des Strukturwandels umfaßt hierbei sowohl die sektorale und regionale Aufteilung der Wirtschaftsleistung auf der Mesoebene als auch einzelwirtschaftliche Güter- und Prozeßstrulkturen sowic Konsummuster und Verhaltenskomponenten im Verbrauchsbereich; darüber hinaus schließt er auch organisatorische Innovationen zur Koordination von Initiativen zum Umweltschutz ein. In diesem Sinn kommt gerade dem ökologischen Strukturwandel eine zentrale Rolle bei umweltpolitischen Strategien zu.

Wie Öko-Effizienz ist auch der Begriff des ökologischen Strukturwandels zunächst nicht eindeutig abgegrenzt. So können im Komponentenmodell die Übergänge zwischen Intensität und Struktur sowie zwischen Struktur und Aktivität fließend sein und von der Betrachtungsebene der Analyse (insbesondere von dem Disaggregationsgrad) abhängen. Zur Konkretisierung und Messung von ökologischem Strukturwandel können vorliegende Indikatoren-Ansätze weiterentwickelt werden, die Ressourcenintensitäten einbeziehen, sich hierauf aber nicht beschränken. Mit dieser Betonung des ökologischen Strukturwandels wird keine Vorauswahl bestimmter umweltpolitischer Instrumente präjudiziert. Der interdisziplinäre Diskurs über Umweltverhalten spricht dafïr, daß eine Kombination unterschiedlicher Maßnahmen den größten Erfolg verspricht.

\section{Anmerkungen}

(1) OECD: Eco-Efficiency. Paris 1998.

(2) DIW/ ISI: Energie-Effizienz-Indikatoren. Untersuchung im Auftrag des Bundesministeriums für Wirtschaft. Berlin, Karlsruhe 1998 (erscheint im Frühjahr 1999 im PhysicaVerlag).

(3) Diekmann, J.: Umwelt, Ökonomik und empirische Sozialforschung. In: Wagner, G./ J. Schupp (Hrsg.): Umwelt und empirische Sozial- und Wirtschaftsforschung. Sonderheft Nr. 165 des DIW, Berlin 1998, S. 187-198.

\section{Der Autor}

Dr. Jochen Diekmann ist wissenschaftlicher Referent im Deutschen Institut für Wirischaftsforschung (DIW), Abteilung Energie, Rohstoffe, Umwelt. Kontakt: DIW, Königin-Luise-Straße 5, 14195 Berlin, Tel. 030/ 89789-693, Fax -200, E-mail: jdiekmann@diw.de 
(c) 20I0 Authors; licensee IÖW and oekom verlag. This is an article distributed under the terms of the Creative Commons Attribution Non-Commercial No Derivates License (http://creativecommons.org/licenses/by-nc-nd/3.o/), which permits unrestricted use, distribution, and reproduction in any medium, provided the original work is properly cited. 